УДК 539.42

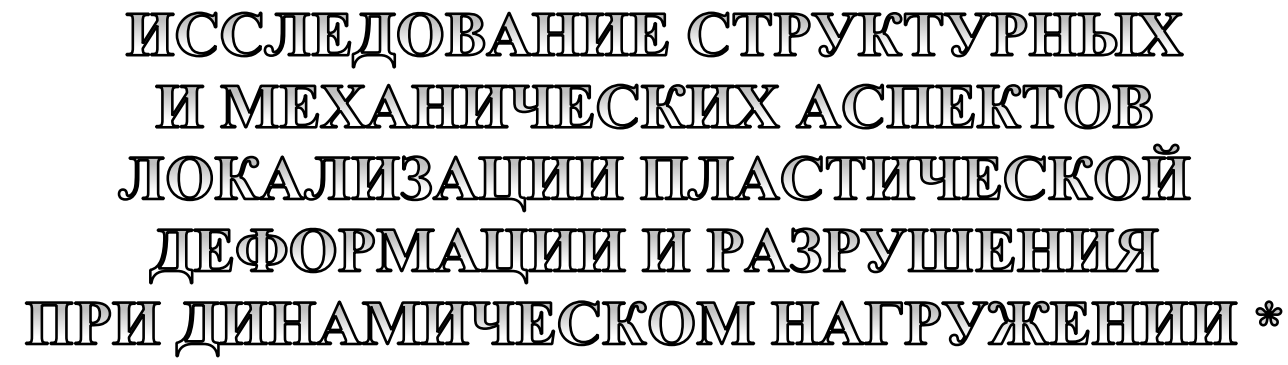

М.А. Соковиков, Институт механики сплошных сред УрО РАН

В.А. Оборин, Институт механики сплошных сред УрО РАН

В.В. Чудинов, Институт механики сплошных сред УрО РАН

Для цитирования:

Соковиков М.А., Оборин В.А., Чудинов В.В. Исследование структурных и механических аспектов локализации пластической десрормации и разрушения при динамическом нагружении // Вестник Пермского федерального исследовательского центра. - 2021. - № 2. - С. 27-33. https://doi.org/10.7242/2658-705X/2021.2.3

Разработана методика и проведены экспериментальные исследования локализации пластической деформации при динамическом кручении образцов из сплавов АМг6, Д16, Сталь 3 на разрезном стержне Гопкинсона-Кольского с применением инфракрасной камеры. Значения температур, определенные in-situ в процессе динамического кручения образцов из сплавов АМг6, Д16, Сталь 3 не превышают $50^{\circ} \mathrm{C}$, что свидетельствует о несущественной роли температурного разупрочнения.

Разработана методика и проведены определения полей деформаций в зоне локализации пластического сдвига на образцах, специально разработанных для изучения локализации пластической деформации из сплавов АМг6, Д16, Сталь 3 при статическом деформировании и динамическом нагружении на стержне Гопкинсона-Кольского с применением системы StrainMaster. Сравнение экспериментально полученных полей деформаций с результатами проведенного численного моделирования дает удовлетворительное соответствие с точностью 20\%.

Сохраненные после экспериментов образцы, специально разработанные для изучения локализации пластической деформации, подвергались микроструктурному анализу с помощью оптического интерферометра-профилометра NewView-5010 с последующей обработкой 3D-данных деформационного рельефа и вычислением масштабного инварианта

* Теоретические исследования осуществлялись в рамках госзадания ПФИЦ УрО РАН (тема № AАAА-А19-119013090021-5). Экспериментальные исследования и численное моделирование осуществлялись при финансовой поддержке гранта РФФИ № 17-41-590149 p_a «Исследование структурных и механических аспектов локализации пластической деформации и разрушения при динамическом нагружении». 
(показателя Хёрста), а также пространственного масштаба области, на которой наблюдается коррелированное поведение микросдвигов. Сравнительный анализ масштабно-инвариантых характеристик статически и динамически нагруженных образцов из сплава Д16 позволил установить значительное увеличение показателя Херста $\mathrm{H} 0,74$ в широком диапазоне пространственных масштабов 0,6-110,8 мкм в отличие от недеформированных образцов (H 0,42 в диапазоне пространственных масштабов 1,9-29,2 мкм). Более содержательные результаты могут быть связаны с изменением как нижнего масштаба, так и верхнего масштаба, что отражает более выраженные черты локализации деформации.

Структура специально разработанных для изучения локализации пластической деформации образцов из сплава АМг6 после динамических испытаний на стержне Гопкинсона-Кольского исследовалась на сканирующем электронном микроскопе. Полученные данные свидетельствует о существенных эволюционных процессах в дефектных подсистемах в процессе динамической деформации.

Ключевые слова: локализачия пластического сдвига, динамическое нагружение, численное моделирование, эволючия дефектной структуры.

Разработана методика экспериментов по изучению локализации пластической деформации при высокоскоростном нагружении методом динамического кручения образцов из алюминиевых сплавов и сталей в условиях чистого сдвига на разрезном стержне Гопкинсона-Кольского с применением инфракрасной камеры с использованием ранее разработанной авторами проекта методики и образцов [1].

С целью отработки методики эксперимента по изучению локализации пластической деформации проведено численное моделирование испытаний по динамическому кручению, определены поля напряжений, деформаций и температур. Разработана и изготовлена оснастка для экспериментов по динамическому кручению образцов для исследования локализованного сдвига.

Проведены эксперименты по изучению локализации пластической деформации при динамическом кручении образцов из сплавов АМг6, Д16, Сталь 3 в условиях чистого сдвига на разрезном стержне Гопкинсона-Кольского с применением инфракрасной камеры с использованием ранее разработанной авторами проекта методики и образцов [1-8]. Значения температур, определенные in-situ, в процессе динамического кручения образцов из сплавов АМг6, Д16, Стали 3. не превышают $50^{\circ} \mathrm{C}$, что свидетельствует о несущественной роли температурного разупрочнения (рис. 1,2$)$.
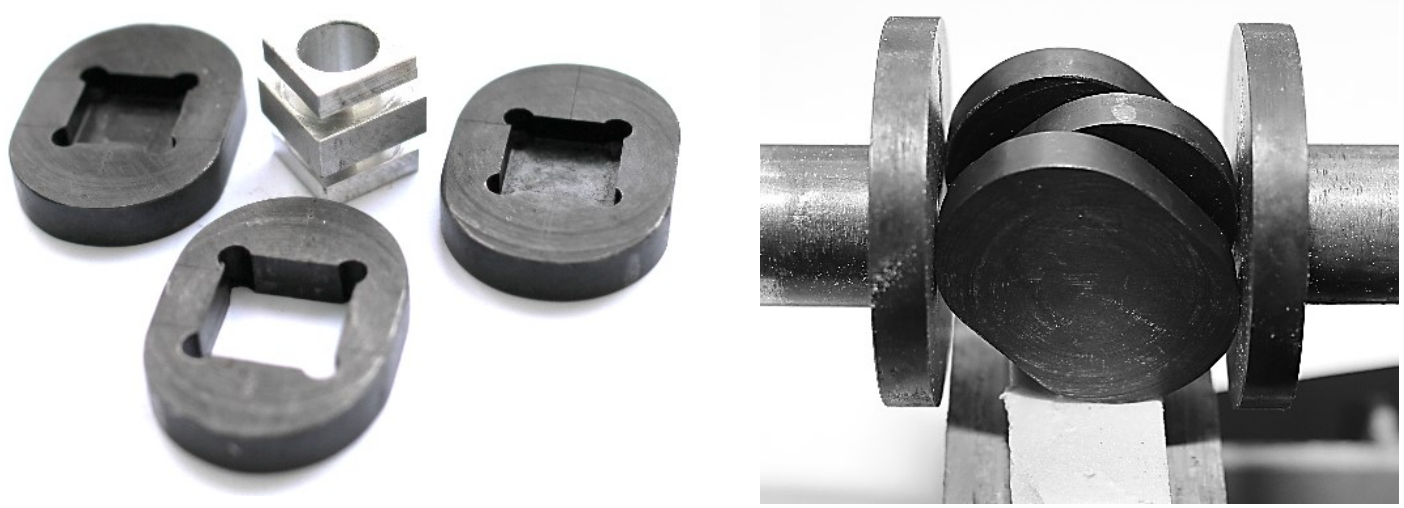

Рис. 1. Образец и оснастка: а-для испьтаний на динамическое кручение; б - установленные между стержнями Гопкинсона-Кольского 


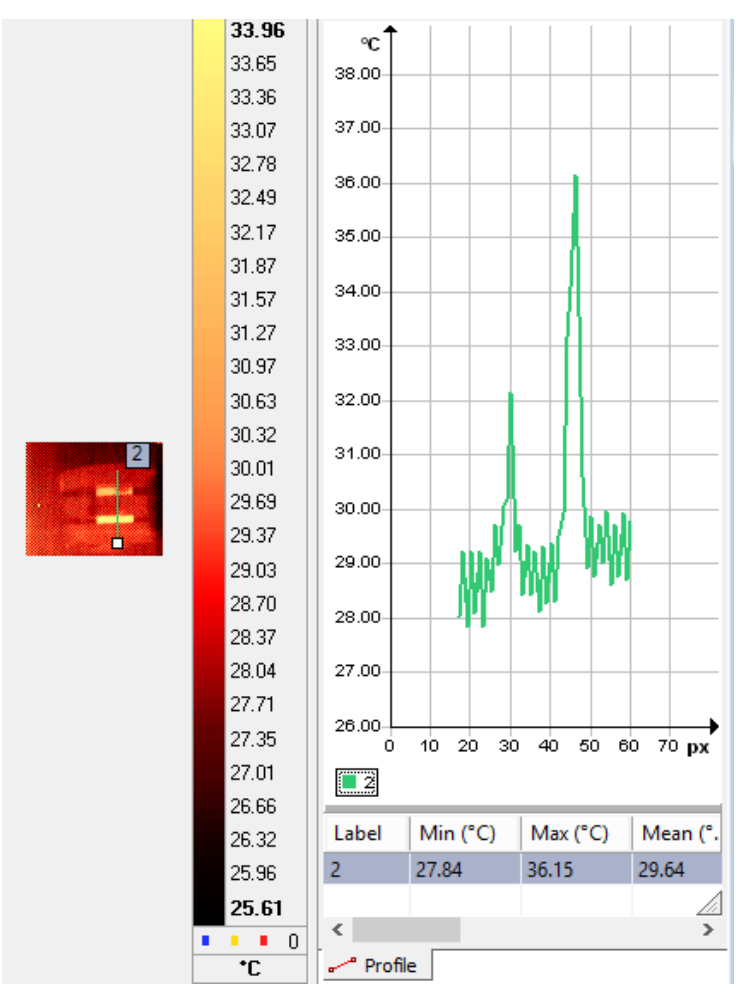

Рис. 2. Образеи в проиессе испытаний в инфракрасных лучах и график зависимости температуры от координаты в выбранный момент времени, материал образиа Д16, максимальная температура $36^{0} \mathrm{C}$

Проведены эксперименты по изучению распределения полей перемещений и деформаций при статическом нагружении на электромеханической испытательной машине «Testometric» образцов специальной П-образной формы из сплавов АМг6, Д16 и Сталь 3 с применением системы неинвазивного измерения формы, деформаций и напряжений StrainMaster.

На основе выводов из статических испытаний проведены динамические испытания образцов специальной П-образной формы из сплавов АМг6, Д16 и Сталь 3 на

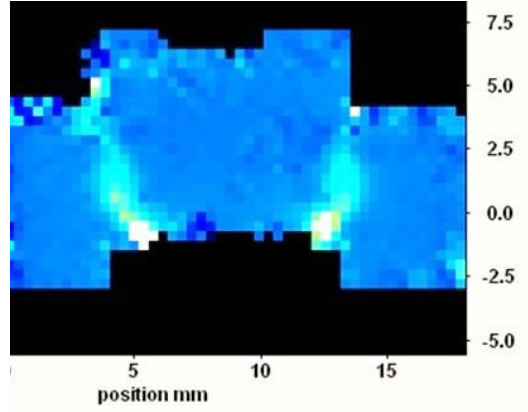

$a$

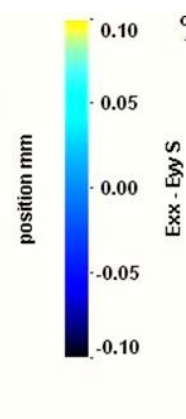

разрезном стержне Гопкинсона-Кольского с применением системы StrainMaster.

Построены поля перемещений и деформаций в П-образных образцах из сплавов АМг6, Д16 и Сталь 3, испытанных при статическом деформировании и динамическом нагружении на стержне Гопкинсона-Кольского. Сравнение экспериментально полученных полей деформаций с результатами проведенного численного моделирования дает удовлетворительное соответствие с точностью $\sim 20 \%$ (рис. 3) [9].

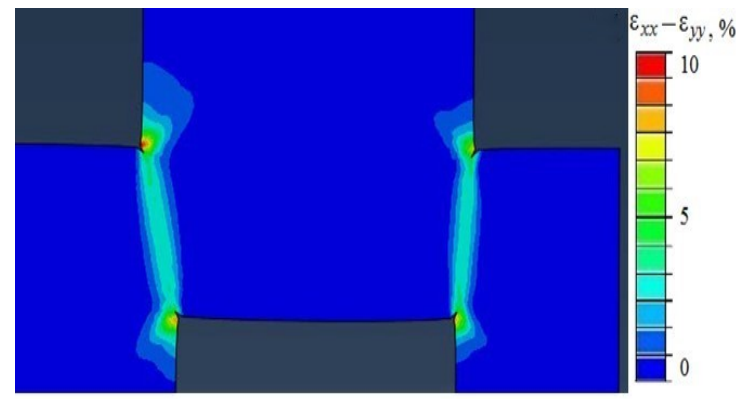

$\sigma$

Рис. 3. Распределение абсолютной величины $\varepsilon_{x x}-\varepsilon_{y y}$ в конечный момент времени при динамическом нагружении образиа специальной формы из сплава АМгб, а-эксперимент, б- численное моделирование 
Сохраненные после экспериментов Побразные образцы подвергались микроструктурному анализу с помощью оптического интерферометра-профилометра NewView-5010 с последующей обработкой 3D-данных деформационного рельефа с последующем вычислением масштабного инварианта (показателя Хёрста) и пространственного масштаба области, на которой наблюдается коррелированное поведение микросдвигов. Определение показателя Хёрста деформационных дефектных структур позволяет дать физическое объяснение универсальности критических явлений применительно к сценариям разрушения различных классов материалов и роли состояния структуры (в том числе формируемой динамическими воздействиями) на «пороговые» характеристики перехода пластически деформированного материала к разрушению $[4,5,9]$. Поверхностный рельеф в области максимальных сдвиговых деформаций деформированных образцов регистрировался с помощью интерферометрапрофилометра высокого разрешения New-View (при увеличении х500) и затем анализировался методами фрактального анализа для определения условий коррелированного поведения многомасштабных дефектных структур. 12 одномерных «срезов» анализировались в пределах каждого «окна» размером 284x213 мкм, обеспечивая представительность данных о структуре рельефа, индуцированного дефектами, с вертикальным разрешением $\sim 0,1$ нм и горизонтальным $\sim 0,45$ мкм.

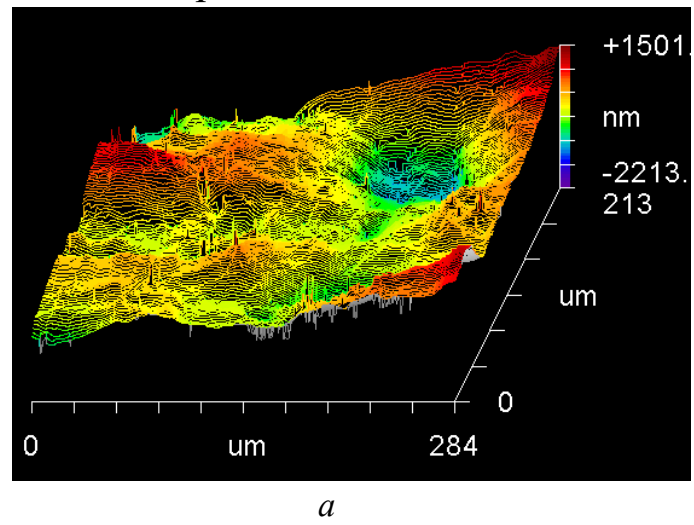

Сравнительный анализ масштабно-инвариантых характеристик статически и динамически нагруженных образцов из сплава Д16 позволил установить значительное увеличение показателя Херста $\mathrm{H} 0,74$ в широком диапазоне пространственных масштабов $\quad 0,6-110,8$ мкм в отличие от недеформированных образцов (H 0,42 в диапазоне пространственных масштабов 1,9-29,2 мкм). Более содержательные результаты могут быть связаны с изменением как нижнего, так и верхнего масштаба, что отражает более выраженные черты локализации деформации. Сами значения пространственных масштабов могут быть использованы для прогнозирования кинетики разрушения при высокоскоростном нагружении. Структурный анализ показал коррелированное поведение ансамбля дефектов, которое может быть классифицировано как структурный переход, обеспечивающий локализацию деформации. Это позволяет рассматривать переход к разрушению локализованным сдвигом как механизм, обусловленный структурными изменениями в материале (рис. 4).

Проведены динамические испытания образцов специальной П-образной формы из Сталь 3 на разрезном стержне Гопкинсона-Кольского с применением инфракрасной камеры. Значения температур определенные in-situ, в процессе динамического деформирования не превышают $80^{\circ} \mathrm{C}$, что свидетельствует о несущественной роли температурного разупрочнения для Сталь 3 в данных условиях нагружения. Был сделан вывод о том, механизм

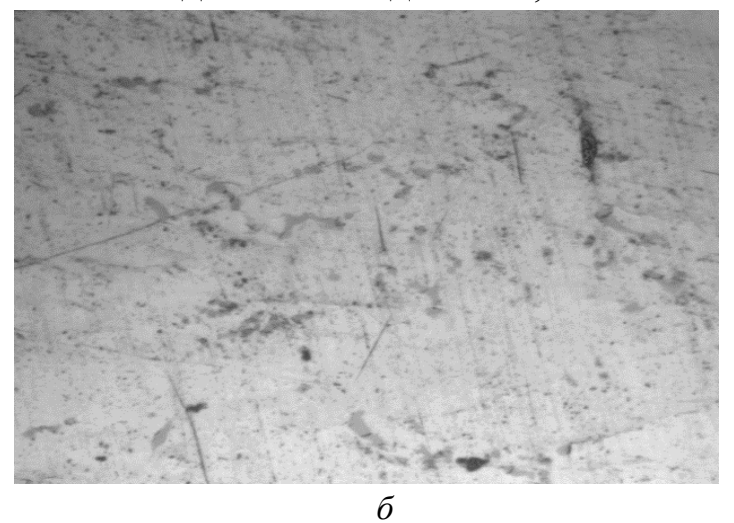

Рис. 4. Образеи из сплава Д16 после испьтаний:а - 3D-образ поверхности после деформирования, боптическое изображение после деформировапния 
локализации пластической деформации для данного материала и реализованных условий нагружения не связан с термопластической неустойчивостью.

Структура П-образных образцов из сплава АМг6 до и после динамических испытаний на стержне Гопкинсона-Кольского исследовалась на сканирующем электронном микроскопе FEI PHENOM G2 ProX при ускоряющем напряжении $15 \kappa В$ и увеличении 1 000-15 000 крат. Исследование структуры показало, что для всех испытанных образцов характерно образование полосовых структур в областях концентрации напряжений. Области локализованного сдвига включают как одиночные кристаллы, так и пакеты кристаллов. В ряде областей системно наблюдались процессы фрагментации полос локализованного сдвига и образование субмикрокристаллического структурного состояния с размерами зерен/субзерен 80-120 нм. Полученные данные свидетельствуют о существенных эволюционных процессах дефектных подсистем в процессе динамической деформации.

К важным особенностям стоит отнести рекристаллизационно-полигонизационные процессы, протекающие перед фронтом образующейся полосы локализованного сдвига, что приводит к образованию мелкокристаллического структурного состояния. Важно отметить, что в том случае, если на пути роста полосы локализованного сдвига находится препятствие в виде включения или полей сегрегаций второй фазы, то рост кристаллов тормозится или полностью прекращается в данном направлении, что приводит к компенсации за счет структурных трансфигураций в близлежащих объемах материала; в таких местах часто наблюдали образование эффекта завихрений деформируемого материала (рис. 5).

Проведена серия испытаний образцов специальной П-образной формы из сплава АМг6 на стержне Гопкинсона-Кольского, аналогичные П-образные образцы были испытаны при статическом нагружении на электромеханической испытательной машине «Testometric». До и после испытаний поверхность образцов исследовалась на оптическом интерферометре-профилометре NewView-5010 с целью определения параметров структурного скейлинга дефектных подсистем. Исследованы микроструктуры испытанных образцов на сканирующем электронном микроскопе FEI PHENOM G2 ProX. Проведено численное моделирование испытаний с использованием модели для эволюции микродефектов. В результате исследований были сделаны выводы о том, что деформирование сплава АМг6 в местах локализации сдвига при статическом и динамическом нагружении реализуется по различным структурным механизмам.

Результаты проведенных исследований указывают на то, локализация пластической деформации в исследуемых

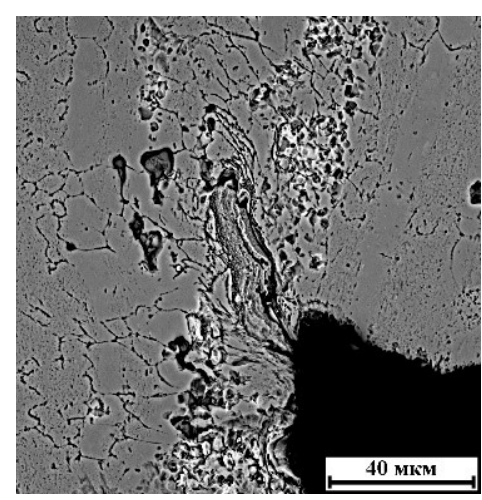

$a$

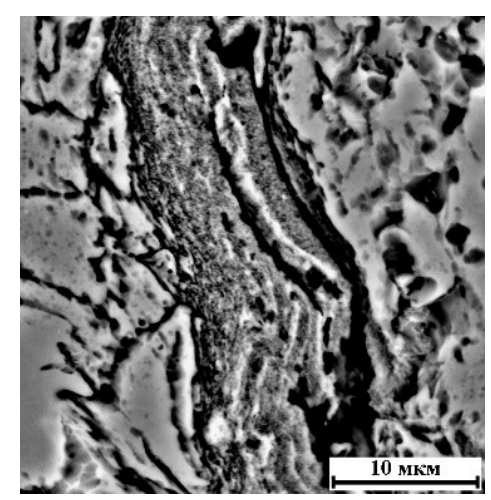

б

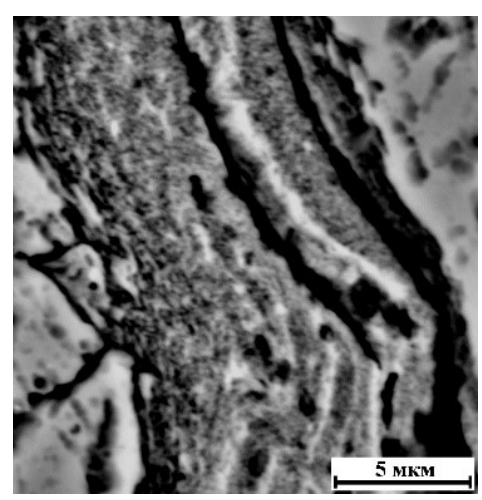

B

Рис. 5. Исследование структуры динамически испытанных П-образных образиов на сканирующем электронном микроскопе FEI PHENOM G2 ProX, Структура локализачии пластической деформации в образие из сплава АМгб: - увеличение х2 000, б-увеличение х8 000, в - увеличение 15000 
материалах и условиях нагружения реа-

лизуется преимущественно в результате

эволюционных процессов в дефектных подсистемах, в условиях коррелиро-

ванного поведения многомасштабных дефектных структур [7-13].

\section{Библиографический список}

1. Наймарк О.Б., Соковиков М.А., Плехов О.А., Уваров С.В., Чудинов В.В., Билалов Д.А., Оборин В.А. Патент РФ № 2584344 от 21.04.2016 г.

2. Соковиков М.А, Билалов Д.А., Чудинов В.В., Уваров С.В., Плехов О.А., Терехина А.И., Наймарк О.Б. Неравновесные переходы в ансамблях дефектов при динамической локализации пластической деформации // Письма в Журнал технической физики. - 2014. - Т. 40, Вып. 23. - С. 82-88.

3. Sokovikov M.A., Bilalov D.A., Oborin V.A., Chudinov V.V., Uvarov S.V., Bayandin Yu.V., Naimark O.B. Structural mechanisms of formation of adiabatic shear bands // Fracture and structural integrity. - 2016. №. 38. - P. 296-304.

4. Билалов Д.А., Соковиков М.А., Чудинов В.В., Оборин В.А., Баяндин Ю.В., Терёхина А.И., Наймарк О.Б. Исследование локализации пластического сдвига в алюминиевых сплавах при динамическом нагружении // Вычислительная механика сплошных сред. - 2015. - Т. 8, № 3. - С. 319-328.

5. Билалов Д.А., Соковиков М.А., Чудинов В.В., Оборин В.А., Баяндин Ю.В., Терёхина А.И., Наймарк О.Б. Численное моделирование и экспериментальное исследование локализации пластической деформации при динамическом нагружении образцов в условиях, близких к чистому сдвигу // Вычислительная механика сплошных сред. - 2017. - Т. 10. - № 1. - С. 103-112.

6. Билалов Д.А., Соковиков М.А., Чудинов В.В. Многомасштабные механизмы локализациипластической деформации при пробивании преград // Деформация и разрушение материалов. - 2017. - № 5. - С. 43-47.

7. Соковиков M.A. Исследование локализации пластической деформации и разрушения при динамическом нагружении методом инфракрасной термографии // Вестник Пермского университета.Физика. - 2018. - Вып. 2(40). - С. 52-57.

8. Соковиков М.A. Исследование локализованного пластического сдвига при динамическом нагружении с применением стержня Гопкинсона-Кольского // Вестник Пермского университета. Физика. - 2019. - № 2. - С. 41-45.

9. Соковиков М.А, Чудинов В.В., Оборин В.А., Уваров С.В., Наймарк О.Б. Изучение механизма разрушения сплавов локализованным сдвигом при динамическом нагружении // Вычислительная механика сплошных сред. - 2019. - Т. 12. - № 3. - С. 301-312.

10. Билалов Д.А., Соковиков М.А., Баяндин Ю.В., Чудинов В.В., Оборин В.А., Наймарк О.Б. Описание эффекта падения прочности сплава АМг6 с ростом температуры при динамическом нагружении // Вестник ПНИПУ. Механика. - 2019. - № 5. - С. 5-14.

11. Sokovikov M., Uvarov S., Simonov M., Oborin V., Chudinov V., Naimark O. The study of mechanical and microstructural aspects of localized shear fracture in metals under dynamic loading // Science Direct Procedia Structural Integrity. - 2019. - Vol. 18. - P. 262-267.

12. Sokovikov M., Simonov M., Chudinov V., Oborin V., Uvarov S., Naimark O. Investigation of Mechanical and Microstructural Aspects of Plastic Shear Localization at Different Types of Dynamic Loading // AIP Conference Proceedings. - 2019. - P. 020346-1-020346-4.

13. Соковиков М.А., Симонов М.Ю., Билалов Д.А., Ю.Н. Симонов Ю.Н., Наймарк О.Б. Локализация пластической деформации всплаве АМг6 при динамическом нагружении // Физическая мезомеханика. - 2020. - Т. 23, № 2. - С. 45-57. 


\title{
INVESTIGATION INTO STRUCTURAL AND MECHANICAL ASPECTS OF LOCALIZATION OF PLASTIC DEFORMATION AND FRACTURE UNDER DYNAMIC LOADING
}

\author{
M.A. Sokovikov, V.A. Oborin, V.V. Chudinov \\ Institute of Continuous Media Mechanics UB RAS
}

\section{For citation:}

Sokovikov M.A., Oborin V.A., Chudinov V.V. Investigation into structural and mechanical aspects of localization of plastic deformation and fracture under dynamic loading // Perm Federal Research Center Journal. - 2021. - № 2. - P. 27-33. https://doi.org/10.7242/2658-705X/2021.2.3

An experimental testing technique was developed to conduct experimental study of plastic strain localization in specimens made of AMg6, D16 and Steel 3 alloys subject to dynamic torsion tests in the split Hopkinson pressure bar device fitted with infrared camera. The temperature values determined insitu during dynamic torsion tests of the specimens made from $\mathrm{AMg} 6$, D16, Steel 3 alloys do not exceed $50^{\circ} \mathrm{C}$, which indicates that temperature softening contributes insignificantly to the process of plastic strain localization. A method was developed and applied for determining the strain fields in the zone of plastic shear localization in the specimens made of AMg6, D16, and Steel 3 alloys, which were specially designed to study plastic strain localization during static deformation and dynamic loading on the split Hopkinson pressure bar using for this purpose the StrainMaster system.

A comparison of the experimentally obtained strain fields with the results of numerical simulation, which was made using the kinetic equations for the evolution of defects of the mesoscale, yields rather good agreement to within $20 \%$.

This study examined post-test specimens specially designed for studying plastic deformation localization. The application of an optical interferometer-profilometer New View - 5010 allowed structural analysis of these specimens, processing of 3D data on the deformation relief and subsequent computation of the scale invariant (Hurst index) and the spatial scale of the zone where the correlated behavior of microshears is observed. The comparison of the scale invariant characteristics of the D16 alloy specimens obtained during static deformation and dynamic loading revealed a significant increase in the Hurst exponent $(\mathrm{H} \sim 0,74)$ over a wide range of spatial scales $(0,6-110,8 \mu \mathrm{m})$ compared to the undeformed specimens $(\mathrm{H} \sim 0,42$ in the range of spatial scales $1,9-29,2 \mu \mathrm{m})$. More meaningful results can be associated with the changes in both lower and upper scales, which indicates pronounced features of the localization of deformation.

The structure of specimens made of AMg6 alloy, specially designed for studying the plastic strain localization after dynamic tests in the split Hopkinson pressure bar, was examined using the scanning electron microscope. The obtained data testify to significant evolutionary processes occurring in the defective subsystems undergoing dynamic deformations.

Keywords: of plastic shear localization, dynamic loading, numerical simulation, evolution of the defect structure.

\section{Сведения об авторах}

Соковиков Михаил Альбертович, кандидат физико-математических наук, старший научный сотрудник лаборатории физических основ прочности, Институт механики сплошных сред УрО РАН - филиал Пермского федерального исследовательского центра УрО РАН («ИМСС УрО РАН»), 614013, г. Пермь, Академика Королева, 1; e-mail: sokovikov@icmm.ru Оборин Владимир Александрович, ведущий инженер лаборатории физических основ прочности, «ИМСС УрО РАН»; e-mail: oborin@icmm.ru

Чудинов Василий Валерьевич, инженер лаборатории физических основ прочности, «ИМСС УрО PAH»; e-mail: chudinov@icmm.ru 